\title{
Serum Proteome Alterations in Patients with Cognitive Impairment after Traumatic Brain Injury Revealed by iTRAQ-Based Quantitative Proteomics
}

\author{
Xin-gui Xiong, ${ }^{1}$ Qinghua Liang, ${ }^{1}$ Chunhu Zhang, ${ }^{1}$ Yang Wang, ${ }^{1}$ Wei Huang, ${ }^{1}$ Weijun Peng, \\ Zhe Wang, ${ }^{2}$ and Zi-an Xia ${ }^{2}$ \\ ${ }^{1}$ Institute of Integrated Medicine, Xiangya Hospital, Central South University, Changsha, Hunan 410008, China \\ ${ }^{2}$ Department of Integrated Traditional Chinese \& Western Medicine, The Second Xiangya Hospital, Central South University, \\ Changsha, Hunan 410011, China
}

Correspondence should be addressed to Weijun Peng; pengweijun1987@gmail.com, Zhe Wang; ericwangzhe@126.com, and Zi-an Xia; xia.zian@csu.edu.cn

Received 3 October 2016; Revised 1 December 2016; Accepted 13 December 2016; Published 30 January 2017

Academic Editor: Lap Ho

Copyright (C) 2017 Xin-gui Xiong et al. This is an open access article distributed under the Creative Commons Attribution License, which permits unrestricted use, distribution, and reproduction in any medium, provided the original work is properly cited.

Background. Cognitive impairment is the leading cause of traumatic brain injury- (TBI-) related disability; however, the underlying pathogenesis of this dysfunction is not completely understood. Methods. Using an isobaric tagging for relative and absolute quantitation- (iTRAQ-) based quantitative proteomic approach, serum samples from healthy control subjects, TBI patients with cognitive impairment, and TBI patients without cognitive impairment were analysed to identify differentially expressed proteins (DEPs) related to post-TBI cognitive impairment. In addition, DEPs were further analysed using bioinformatic platforms and validated using enzyme-linked immunosorbent assays (ELISA). Results. A total of 56 DEPs were identified that were specifically related to TBI-induced cognitive impairment. Bioinformatic analysis revealed that a wide variety of cellular and metabolic processes and some signaling pathways were involved in the pathophysiology of cognitive deficits following TBI. Five randomly selected DEPs were validated using ELISA in an additional 105 cases, and the results also supported the experimental findings. Conclusions. Despite limitations, our findings will facilitate further studies of the pathological mechanisms underlying TBI-induced cognitive impairment and provide new methods for the research and development of neuroprotective agents. However, further investigation on a large cohort is warranted.

\section{Introduction}

Traumatic brain injury (TBI) is a major public health concern that affects $12 \%$ of the general population and results in high rates of death and disability worldwide [1]. Approximately $65 \%$ of moderate-to-severe TBI patients suffer from long-lasting cognitive deficits, including deficits in memory capacity, attention, executive functions, and general cognitive intelligence $[2,3]$. These deficits impose a personal and economic burden that is difficult to quantify. Despite significant efforts, the pathologies underlying TBI-induced cognitive impairment are poorly understood, and effective disease-modifying treatment strategies are lacking [4, 5]. Thus, investigation of alternative disease mechanisms of
TBI-induced impairment are required to better understand its pathophysiology and to yield effective therapeutic agents [4].

Due to the complexity and heterogeneity of TBI-induced cognitive impairment, it is likely that multiple candidate proteins present in networks are perturbed, leading to the spectrum of cognitive symptoms. Currently, with the advent of quantitative proteomic technologies using an isobaric labelling strategy, it has become possible to quantify several proteins in a single experiment for the comparative study of global protein regulation across various biological samples, and this method has been widely applied to elucidate disease mechanisms [6-9]. Therefore, this method represents an exciting new approach that can help to address the 
complex pathology of TBI-induced cognitive impairment. A novel quantitative proteomic technology, isobaric tagging for relative and absolute quantitation (iTRAQ), has recently become a powerful tool to characterize protein expression during different physiological and pathological states. In particular, several studies have successfully applied this novel quantitative proteomic technology to identify the biomarkers for TBI in rats with diffuse axonal injury [10], patients with elevated intercranial pressure [11], and an ApoE mouse model [12].

However, none of the previous studies applied a quantitative proteomic technology to identify global protein changes and pathways perturbed in post-TBI cognitive impairment using clinical samples. In this study, an iTRAQ-based quantitative proteomics approach was adopted to identify and quantity the differentially expressed proteins (DEPs) in serum samples from TBI patients with cognitive impairment. In addition, DEPs were further analysed by bioinformatic platforms and validated by enzyme-linked immunosorbent assays (ELISA). These findings will further the understanding of the pathophysiological mechanisms underlying post-TBI cognitive impairments.

\section{Material and Methods}

2.1. Ethics Statement. All protocols involving the use of human subjects were reviewed and approved by the Ethics Committee of Central South University, Changsha, China (Grant no. 201404366), and all experiments were performed in accordance with the Declaration of Helsinki. Written informed consent was obtained from all participants (or their legal guardians) enrolled in this study.

2.2. Study Subjects. All participants presented at the Brain Trauma Specialist Department, Department of Encephalopathy of the National Key Specialty, and the Health Centre of the Xiangya Hospital, Central South University, Changsha, China, between February 2014 and December 2014. The subjects were divided into three groups: healthy controls (HC group), TBI patients without cognitive deficits (negative group), and TBI patients with cognitive deficits (positive group).

As described in our previous study [13], TBI patients were screened to meet the following inclusion criteria: (i) age > 18 and <65 years; (ii) a history of moderate-tosevere TBI (defined as an initial Glasgow Coma Scale (GCS) score of 12 or less); (iii) 1 to 6 months after initial injury; (iv) being without various types of extracranial injuries; and (v) no anticipated/pending neurosurgical operative intervention.

Individuals were excluded if they met any of the following criteria: (i) serious conditions causing mental disability prior to the TBI, such as a developmental handicap (Down's syndrome), residual disability after previous TBI, confirmed dementia, or serious chronic mental illness (schizophrenia, psychosis, or well-confirmed bipolar disorder); (ii) severe renal or hepatic impairment; (iii) uncontrolled cardiovascular disease; (iv) a current history of severe abuse of drugs or alcohol; and (v) being pregnant or lactating.
When TBI patients were recruited into study, the overall level of cognitive and behavioural functioning of them was assessed using the Rancho Los Amigos Scale (RLAS, also referred to as "Rancho" or the "Levels of Cognitive Functioning Scale") by two doctors $[14,15]$. TBI patients who obtained scores ranging from Level 1 to Level 8 were classified into the positive group, and those with a level of cognitive and behavioural functioning higher than Level 8 were classified into the negative group.

In addition, the healthy control group was composed of healthy volunteers with no current or previous lifetime history of neurological diseases or systemic medical illness. Healthy controls were matched with TBI patients for age and gender. The demographic and clinical chemistry characteristics of enrolled subjects are shown in Table 1.

2.3. Sample Collection and Preparation. The subjects fasted for at least 12 hours before blood was drawn. The blood samples were obtained specifically for the purpose of this study and were coded to maintain anonymity. Relevant medical data were recorded and coded to match the extracted blood samples. A $3 \mathrm{~mL}$ blood serum sample was collected from each enrolled subject. The serum samples were placed in Eppendorf tubes without anticoagulant at $4^{\circ} \mathrm{C}$ and allowed to stand for $1 \mathrm{~h}$. The sample was centrifuged at $3000 \mathrm{~g}$ at $4^{\circ} \mathrm{C}$ for $15 \mathrm{~min}$. The supernatant consisted of the total serum proteins. Then, the sample was divided into $0.5 \mathrm{~mL}$ aliquots and stored at $-80^{\circ} \mathrm{C}$ in a refrigerator for future use.

Pooled serum samples were generated by combining equal volumes of the 15 individual plasma samples from each group $(n=16)$. The high-abundance proteins of each serum pool were depleted using ProteoMiner Protein Enrichment Kits (Bio-Rad, USA) according to the manufacturer's instructions. The protein concentration was determined using a Bradford Protein Assay Kit (Amesco, Ohio, USA) [16].

2.4. iTRAQ-Based Quantitative Proteomics Analysis. As described in our previous study [17], the analytic processes were performed by the Beijing Protein Innovation Co., Ltd., Beijing, China, and included protein digestion, iTRAQ labelling, strong cation exchange (SCX) fractionation, LC-MS/MS analysis, protein identification, and protein quantitation.

Briefly, trypsin digestion and iTRAQ labelling were performed according to the manufacturer's protocol (Applied Biosystems). First, $50 \mu \mathrm{g}$ of protein from each pooled sample was reduced, alkylated, and digested overnight at $37^{\circ} \mathrm{C}$ with trypsin. Then, according to the iTRAQ Reagent Multiplex Kit (Applied Biosystems) protocol, the tryptic peptide solution of each sample was labelled with iTRAQ reagents as follows: control group, iTRAQ reagent 113; negative group, iTRAQ reagent 114; positive group, iTRAQ reagent 115 . The tryptic peptide samples were labelled, mixed, and dried before further analyses.

The mixed peptides were fractionated by SCX chromatography on an ultimate high-performance liquid chromatography (HPLC) system (Shimadzu, Kyoto, Japan) with an SCX column (Luna SCX 100A, Phenomenex). Based on the SCX chromatograms, 10 SCX fractions were collected along the 
TABLE 1: Demographic characteristics of the enrolled participants.

\begin{tabular}{|c|c|c|c|}
\hline & Positive & Negative & $\mathrm{HC}$ \\
\hline Number & 51 & 51 & 51 \\
\hline Female/male & $20 / 31$ & $22 / 29$ & $24 / 27$ \\
\hline Age & $35.7 \pm 12.6$ & $36.9 \pm 14.8$ & $38.6 \pm 11.5$ \\
\hline Disease duration (days) & $45.10 \pm 3.75$ & $49.50 \pm 4.79$ & 1 \\
\hline \multicolumn{4}{|l|}{ Cause of TBI } \\
\hline Transport accidents & 35 & 40 & l \\
\hline Fall & 10 & 8 & l \\
\hline Assaults & 2 & 1 & l \\
\hline Others & 4 & 2 & l \\
\hline $\begin{array}{l}\text { GCS score at admission } \\
\text { median (IQR) }\end{array}$ & $7(4-9)$ & $8(4-10)$ & l \\
\hline Moderate/severe ${ }^{(1)}$ & $31 / 20$ & $26 / 25$ & l \\
\hline \multicolumn{4}{|l|}{ Neurosurgery $^{2}$} \\
\hline No/yes & $28 / 23$ & $31 / 20$ & l \\
\hline Multiple ICD-10 diagnosis (S06) & 43 & 44 & l \\
\hline
\end{tabular}

Note: IQR, interquartile range. ${ }^{(1)}$ Moderate/severe indicates the classification of TBI according to GCS score at admission. ${ }^{2}$ Neurosurgery. "Yes" indicates the patients who underwent neurosurgical operative intervention at admission, whereas "No" indicates those who did not.

gradient. Each SCX fraction was dried, dissolved, and then analysed on a reverse-phase liquid chromatography column (Strata-X C18 column, $5 \mu \mathrm{m}, 300 \mathrm{~A}, 100 \mathrm{~mm} \times 75 \mathrm{~mm}$, Phenomenex).

Mass spectrometry (MS) analysis of the iTRAQ-labelled samples was performed on a Q Exactive LC-MS/MS (ThermoFisher Scientific, Waltham, MA, USA) mass spectrometer. Sequences for the peptide and reporter ions were generated to identify the protein from which the peptide originated. To minimize the effect of experimental variation, three independent MS/MS runs were performed for each sample.

Proteome Discoverer Software (Thermo Scientific version 1.3) was used for the data acquisition and quantification. The data sifted by Proteome Discoverer were used to identify proteins using Mascot (version 2.3.0, Matrix Science, London, UK) software and the Uniprot-rat database (http://www.uniprot.org/). The quantitative result of the peptide was the ratio of the signal intensity value of the reference sample (normal sample) label to the signal strength values of other labels. The protein quantitative ratio was calculated as the median of all peptide ratios. The final quantitative result was normalized to the median ratio of each label. The fold change of the DEPs was calculated as the average value from the protein iTRAQ ratios. The DEPs were identified according to the following criteria: the minimum fold change was \pm 1.2 , the difference was statistically significant $(P<0.05)$, and no less than one peptide within the $95 \%$ confidence interval was included [18].

2.5. Bioinformatic Analysis. As described in a previous study [19], a list containing all DEPs identified previously was submitted to the Gene Ontology Consortium, and PANTHER database analysis tools were applied for functional annotation and enrichment analysis [20]. Pathway analysis was performed using the KEGG database. Predicted proteinprotein interactions (PPIs) were generated and visualized using STRING software [21]. $P$ values and Benjaminicorrected $P$ values less than 0.05 were considered significant.

2.6. ELISA Validation. As described in our previous study [22], the serum levels of selected DEPs were measured using an ELISA quantitation kit (USCN Life Sciences, Wuhan, China) following protocols recommended by the manufacturer.

2.7. Statistical Analyses. All data are expressed as the means \pm SE. One-way ANOVA was used to compare the differences between the groups. All statistical analyses were conducted using the SPSS (version 22.0, Chicago, IL). $P$ values $<0.05$ were considered to indicate statistically significant differences.

\section{Results}

3.1. Identification and Relative Quantification of Dysregulated Proteins. Using iTRAQ-based quantitative proteomics, a total of 331,259 MS/MS spectra were obtained, of which 40,273 were matched. Then, 48,350 PSMs were assigned to 3079 peptides after 1\% FDR was applied. Through this strategy, 359 proteins were identified for further study. Of the 359 proteins, we identified 50 DEPs in the positive and negative subjects, including 23 upregulated and 27 downregulated proteins. Meanwhile, 108 DEPs were identified in the positive and control subjects, including 54 upregulated and 54 downregulated proteins. Additionally, 87 DEPs were identified in the negative and control subjects, including 37 upregulated and 40 downregulated proteins. As shown in Figure 1, of these dysregulated proteins, 56 DEPs were specific to TBI-induced cognitive impairment (Figure 1, Table 2). 


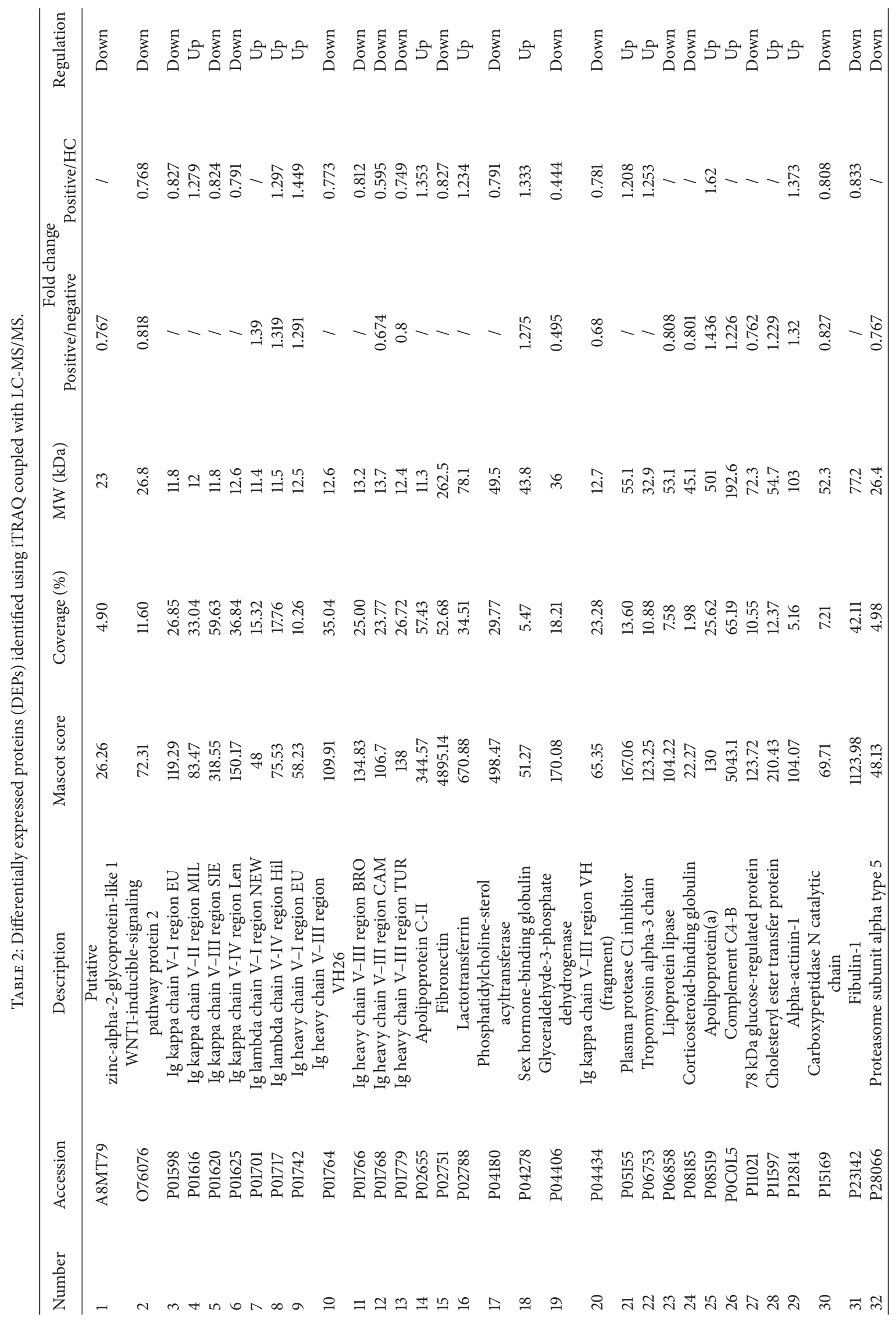




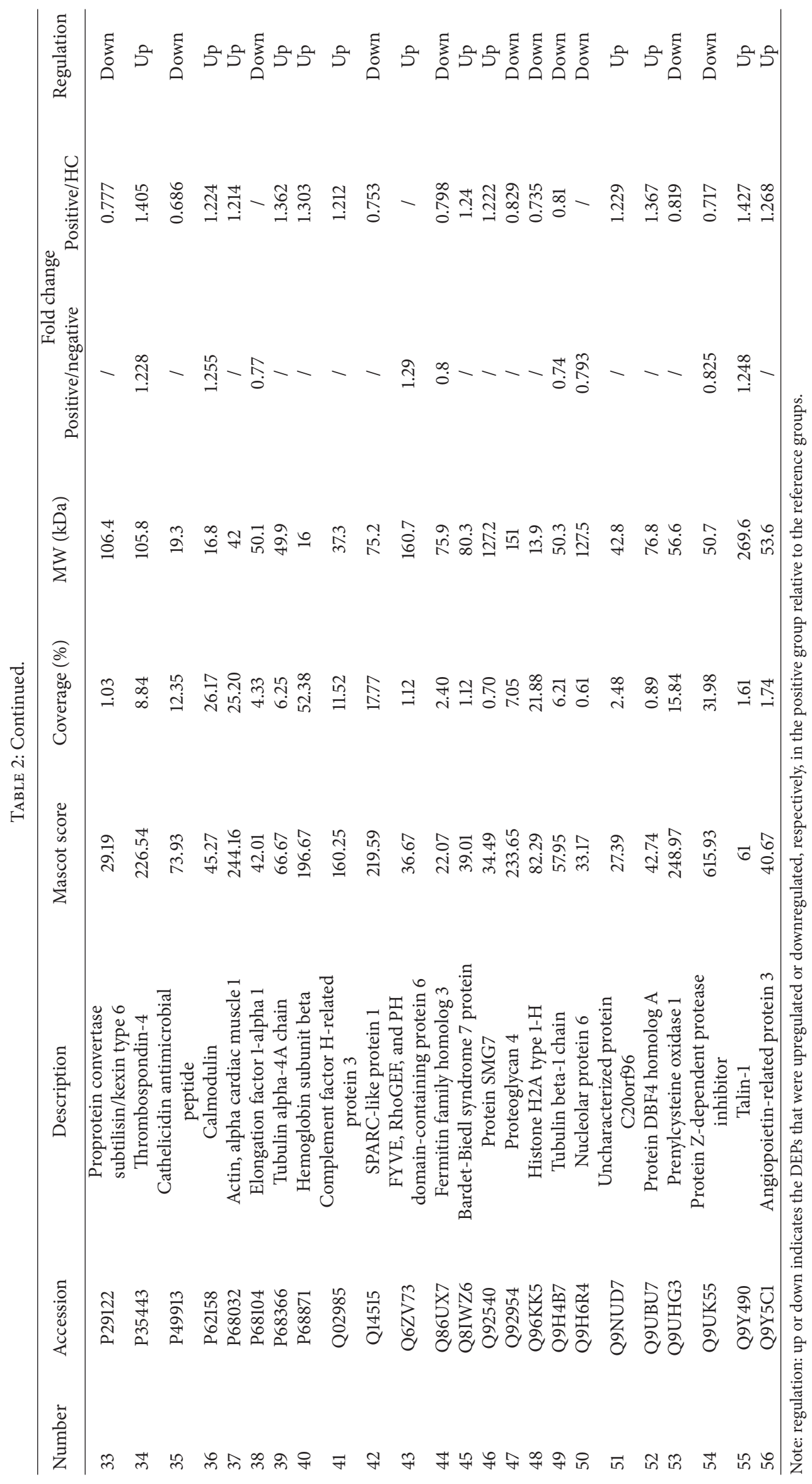




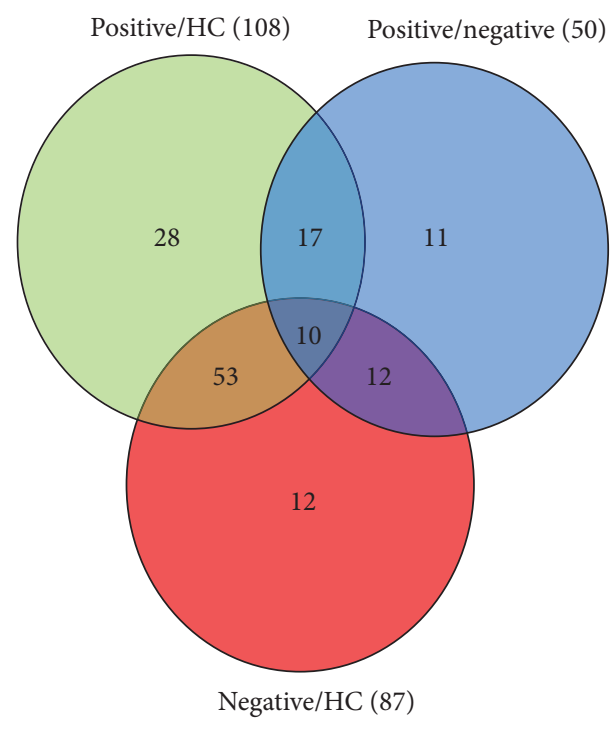

FIGURE 1: Venn diagram showing the number of differentially expressed proteins (DEPs) and their overlap. The results indicated 108 proteins showed differential expression in the positive versus healthy control (HC) groups (green cycle), 50 proteins in the positive versus negative groups (blue cycle), and 87 proteins in the negative versus HC groups (red cycle). A total of 56 DEPs which included 28 DEPs in the positive versus negative comparison, 11 DEPs in the positive versus negative comparison, and 17 DEPs in the overlapping regions between both comparisons (positive versus negative and positive versus negative) were specific to the positive group.

3.2. Functional Classification of DEPs. To gain insight into the biological changes in TBI patients with cognitive impairment, the DEPs were categorized according to the following Gene Ontology (GO) classes: biological process, molecular function, and cellular components (Figure 2). In the biological processes class, most of the DEPs were mainly associated with cellular component organization or biogenesis (GO:0071840, 11.5\%), cellular processes (GO:0009987, $28.7 \%$ ), localization (GO:0051179, 10.3\%), and metabolic processes (GO:0008152, 16.1\%) (Figure 2(a)). In the molecular function group, the DEPs were mainly found to be involved in binding (GO:0005488, 40.5\%), structural molecule activity (GO:0005198, 14.3\%), and catalytic activity (GO:0003824, $40.5 \%$ ) (Figure 2(b)). In the cellular components class, the DEPs were mainly located in macromolecular complexes (GO:0032991, 11.6\%), the cellular region (GO:0044464, $27.9 \%$ ), organelles (GO:0043226, 23.3\%), and the extracellular region (GO:0005576, 25.6\%) (Figure 2(c)).

To determine whether the DEPs were enriched in certain groups, we employed the PANTHER Overrepresentation Test and used the Bonferroni correction for multiple comparisons. As shown in Figure 2(d), we found that the DEPs involved in vesicle-mediated transport (GO:0016192) were the most significantly enriched group in the biological process group $(P=0.000007)$, the DEPs involved in enzyme regulatory activity (GO:0030234) were the most significantly enriched group in the molecular function group $(P=0.000001)$, and the DEPs involved in the extracellular region (GO:0005576) were the most significantly enriched group in the cellular component group $(P<0.00001)$.

3.3. KEGG Pathway Analysis. Because we were interested in the signaling pathways enriched in DEPs, a KEGG pathway analysis was performed. As shown in Table 3, 16 significantly enriched pathways were found, including pathways involved in Alzheimer's disease (AD) (ko05010, $P=0.015594)$. Additionally, glyceraldehyde-3-phosphate dehydrogenase (GAPDH), calmodulin (CaM), and lipoprotein lipase (LPL) were involved in the $\mathrm{AD}$ pathways (Figure S1 in Supplementary Material available online at https://doi.org/10.1155/2017/8572509).

3.4. Protein-Protein Interaction (PPI) Analysis of the DEPs. The 56 DEPs identified in the current study were submitted to STRING to assess the PPI networks (Figure 3). Of the 56 identified DEPs, 45 were mapped in PPI networks, 29 were interconnected, and 16 proteins did not show any type of connection at the selected confidence level (STRING score $=0.4$ ). In addition, we found that CaM (CALM1, CALM2, and CALM3), LPL, GAPDH, tubulin alpha-4A (TUBA4A), and actin alpha cardiac muscle 1 (ACTC1) were located in a network hub and exhibited a complex relationship with the other proteins.

3.5. Verification of the DEPS Using ELISA. Based on the results of the bioinformatic analysis and the correlations with disease pathogenesis, five candidate DEPs, namely, GAPDH, CaM, apolipoprotein(a) (APO(a)), thrombospondin-4 (THBS4), and Talin-1 (TLN1), were selected for validation in an additional 105 cases using ELISA. These cases included 35 TBI patients with cognitive impairment, 35 TBI patients without cognitive impairment, and 35 healthy controls.

As shown in Figure 4, consistent with the data obtained in the proteomic studies, the results revealed significantly increased serum CaM, APO(a), THBS4, and Talin-1 levels $(P=0.007, P=0.018, P=0.0019$, and $P=0.006$, resp. $)$ and significantly reduced GAPDH levels $(P=0.025)$ in the positive group compared to the negative group. In addition, significant differences in GAPDH, CaM, APO(a), THBS4, and Talin-1 levels were also observed between the positive and control groups $(P=0.007, P<0.005, P=0.001, P=0.006$, and $P=0.001$, resp.).

\section{Discussion}

In our study, using an iTRAQ-based quantitative proteomic approach, a total of 56 DEPs were found which displayed quantitative changes unique to TBI patients with cognitive deficits relative to healthy controls and TBI patients without cognitive deficits. Of these DEPs, 30 were downregulated, including LPL and GAPDH, and 26 were upregulated, including $\mathrm{APO}(\mathrm{a})$, THBS4, and CaM.

According to the bioinformatic analysis, the 56 DEPs were suggested to be involved in a wide variety of cellular and metabolic processes, including immunity and inflammation, transportation of important regulatory biomolecules, 


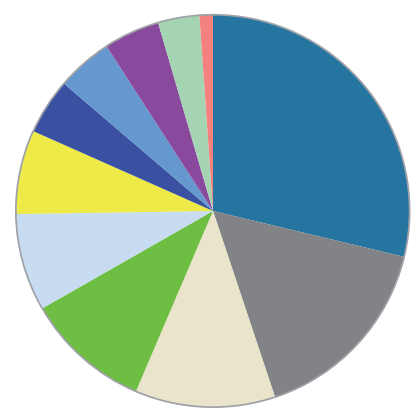

- Cellular process (GO:0009987)

- Metabolic process (GO:0008152)

$\square$ Cellular component organization or biogenesis (GO:0071840)

Localization (GO:0051179)

$\square$ Biological regulation (GO:0065007)

$\square$ Immune system process (GO:0002376)

- Response to stimulus (GO:0050896)

- Developmental process (GO:0032502)

- Biological adhesion (GO:0022610)

$\square$ Multicellular organismal process (GO:0032501)

- Reproduction (GO:0000003)

(a) GO biological process

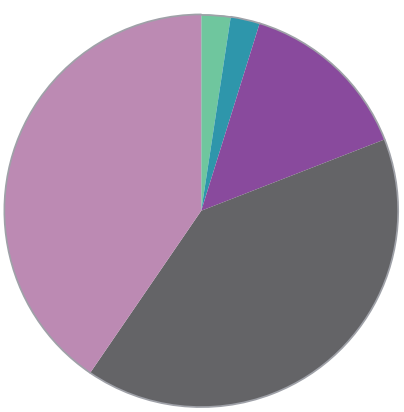

Translation regulator activity (GO:0045182)

- Transporter activity (GO:0005215)

- Structural molecule activity (GO:0005198)

- Binding (GO:0005488)

- Catalytic activity (GO:0003824)

(b) GO molecular function

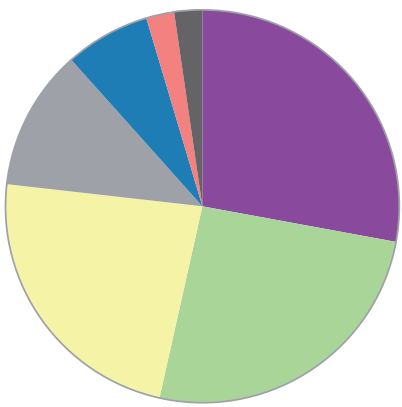

Cell part (GO:0044464)

Extracellular region (GO:0005576)

Organelle (GO:0043226)

Macromolecular complex (GO:0032991)

- Membrane (GO:0016020)

- Synapse (GO:0045202)

- Extracellular matrix (GO:0031012)

(c) GO cellular component

FIgURE 2: Continued. 


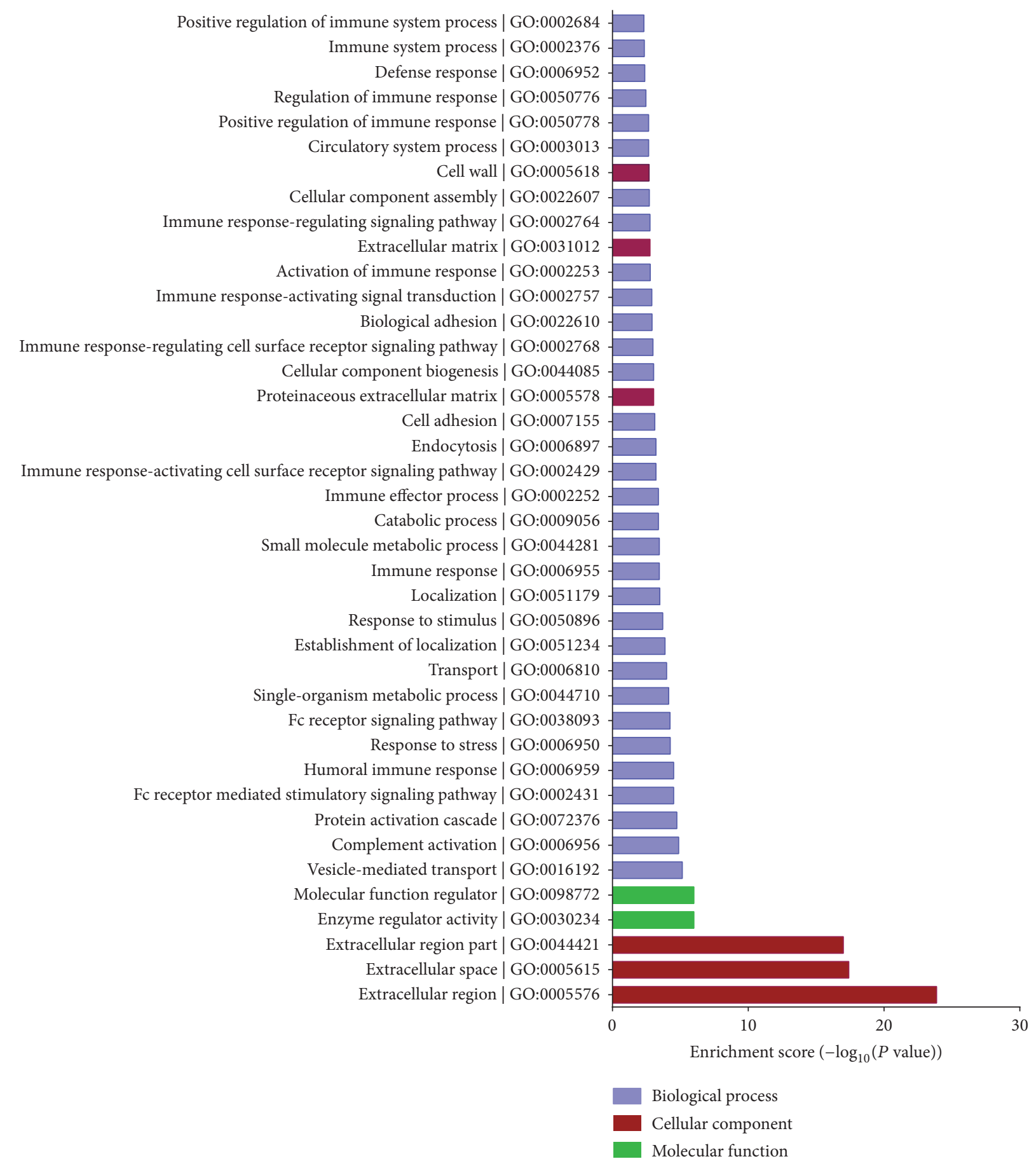

(d)

FIGURE 2: GO analysis of the differentially expressed proteins (DEPs). All identified proteins were functionally annotated in GO database according to their biological process (a), molecular function (b), and cellular component (c). In addition, the GO term enrichment analysis was conducted, and the significantly enriched categories $(P<0.05)$ were recorded $(d)$.

blood coagulation, and other cell processes, and a sizeable group of significantly differentiated pathways with important biological functions. Moreover, the results of PPI analysis indicated that TBI-induced cognitive impairment is a multifactorial process of pathological progress that involves various proteins that interact with each other including the SPARCL1-CALM-ACTC1-TUBA4A-GAPDHLPL-SHBG-SERPINA6 network. In addition, the ELISA validation results confirmed the proteomics analysis findings to some extent.

Interestingly, our results revealed that $\mathrm{AD}$ signaling pathways (including LPL, GAPDH, and CaM) might play an 


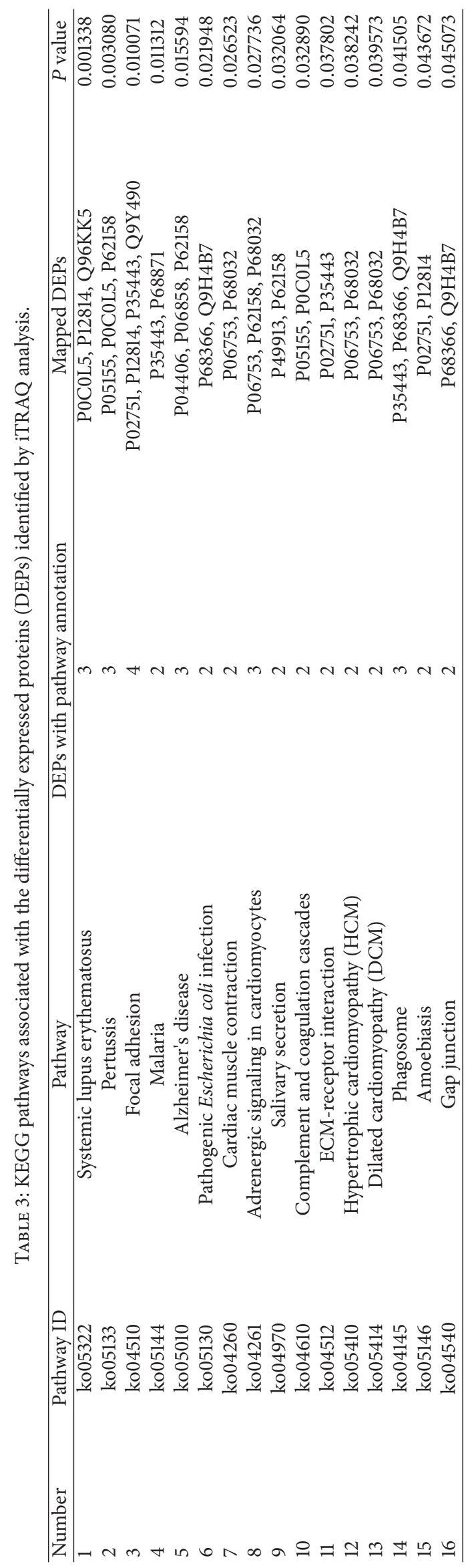




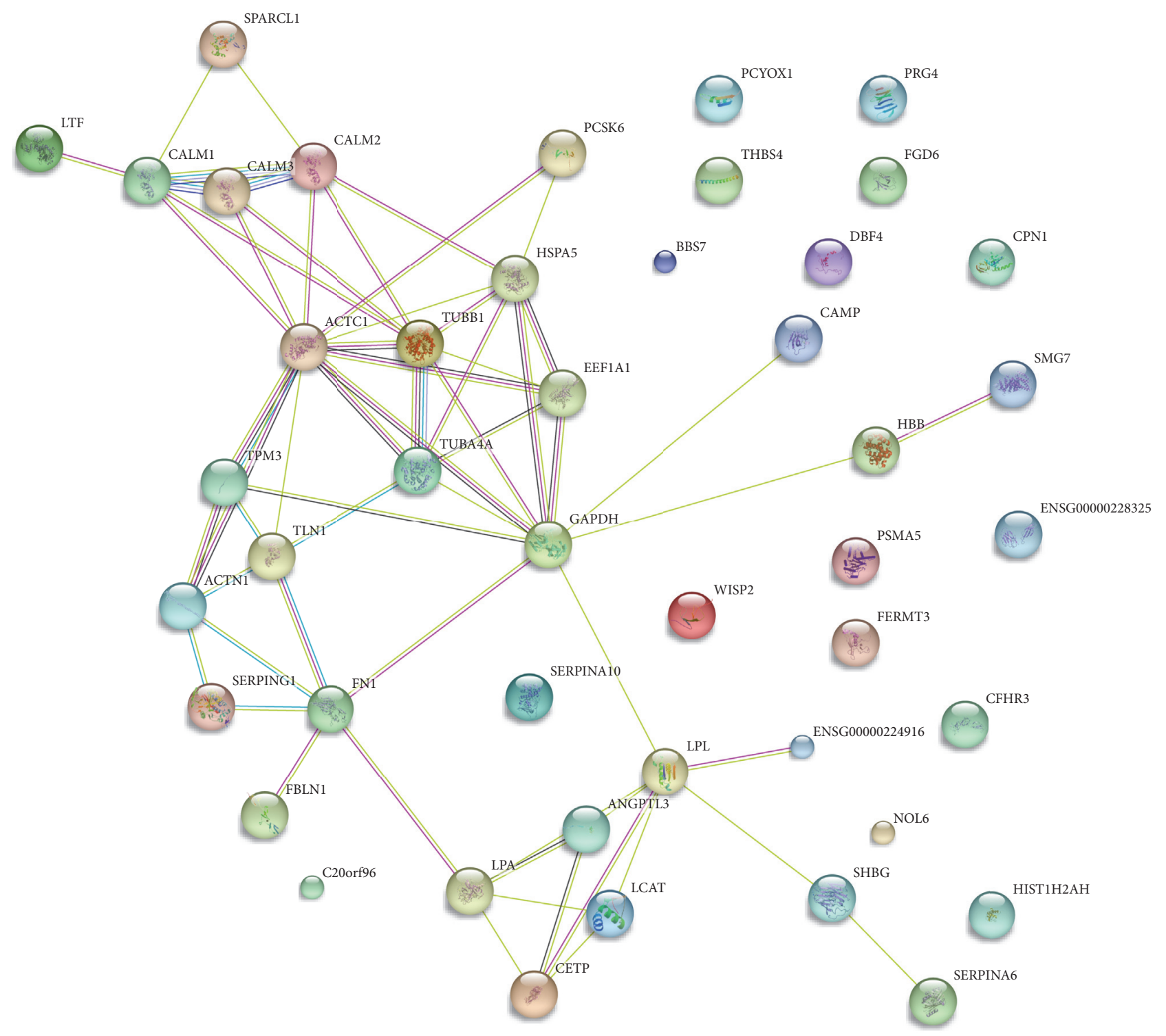

FIGURE 3: Protein-protein interactions for the differentially expressed proteins identified using iTRAQ-based proteomics were analysed with STRING V10.0. In the network, the proteins are represented as nodes. The colors of the lines connecting the nodes represent different evidence types for the protein linkage.

important role in the pathophysiology of post-TBI cognitive impairments. Numerous epidemiological studies have indicated that TBI can increase the risk of developing AD, which is the most common form of dementia [23, 24]. Our present findings provide further evidence of the association between $\mathrm{TBI}$ and $\mathrm{AD}$ from the perspective of serum protein expression profiling.

As a classical glycolytic enzyme, GAPDH was validated by ELISA as being significantly downregulated in the TBI patients with cognitive impairment. In addition, GAPDH has been suggested to have high affinity for AD-associated proteins, including $\beta$-amyloid, $\beta$-amyloid precursor protein, and tau [25], and to be involved in the NO/GAPDH/Siah-1 apoptotic cell death cascade [26], particularly in neuronal cell death associated with neurodegenerative diseases [25, 27].
CaM, the major intracellular $\mathrm{Ca}^{2+}$ ion-binding protein, was significantly upregulated in the TBI patients with cognitive impairment. As a primary $\mathrm{Ca}^{2+}$ signal transducer, CaM responds to cytosolic $\mathrm{Ca}^{2+}$ fluxes by binding to and regulating the activity of target CaM-binding proteins (CaMBPs) [28]. Several experimentally verified CaMBPs are involved in the formation of amyloid- $\beta$ plaques, including amyloid- $\beta$ protein precursor, $\beta$-secretase, presenilin-1, and ADAM10 [29]. Previous studies have demonstrated that LPL is highly expressed in the pyramidal cells of the hippocampus and involved in the pathogenesis of dementia [30]. Our study has shown that the TBI patients with cognitive deficits had lower serum LPL levels, which is consistent with previous reports that LPL-deficient mice display memory impairments [31]. Moreover, LPL has been suggested to be associated with 


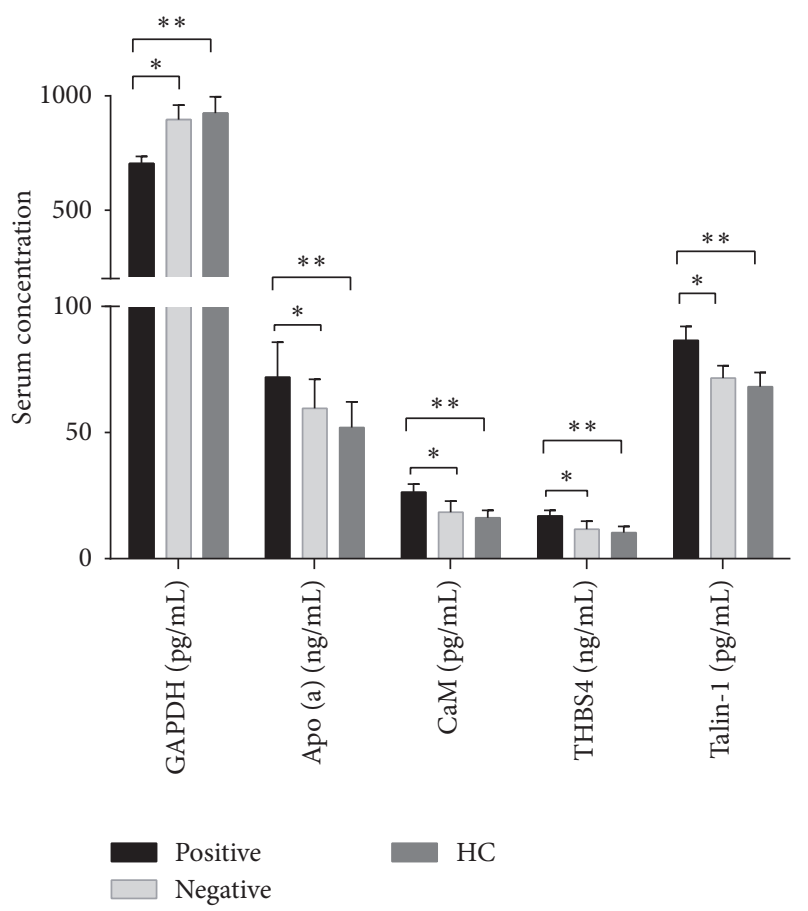

FIgURE 4: Serum proteins levels among the positive, negative, and HC group. A $P$ value less than 0.05 indicates statistical significance using the Mann-Whitney $U$-test. ${ }^{*} P<0.05$ and ${ }^{* *} P<0.01$.

neurite pathology, and its levels are markedly reduced in the dentate gyrus of AD brains [32]. Thus, its role in post-TBI cognitive impairments is worthy of further investigations.

Additionally, we found that lipid metabolism-associated DEPs, including ApoC-II, Apo(a), cholesteryl ester transfer protein (CETP), and LPL, were significantly altered in the TBI patients with cognitive impairment. It has been suggested that dysregulated lipid metabolism may play an important role in the pathophysiology of post-TBI cognitive impairments. As the main constituent of lipoprotein(a), Apo(a) was validated using ELISA as being significantly upregulated in the TBI patients with cognitive impairment. It has been demonstrated that Apo(a) may be involved in the pathology of dementia by participating in amyloidogenesis and playing a role in neuronal maintenance [33]. Moreover, Apo(a) can alter ApoE isoform metabolism, which suppresses changes related to the development of dementia. ApoC-II and CETP were upregulated in the TBI patients with cognitive impairment. ApoCII is normally bound to chylomicrons and very-low density lipoproteins and has been found to play an important role in activation of LPL activity [34]. CETP is a key player in lipid metabolism and catalyses the transfer of cholesteryl esters from high-density lipoprotein particles to triglyceride-rich lipoproteins in exchange for triglycerides. In addition, CETP is linked to cerebral cholesterol metabolism and associated with cognitive function [35].

THBS4 and Talin-1 were also both validated using ELISA as being significantly upregulated in the TBI patients with cognitive impairment. In contrast, accumulated data indicate that THBS4 could not only regulate synapse formation but also play an important role in neurite and axon outgrowths [36]. Furthermore, THBS4 has been suggested to play a role in regulating protective astrogenesis from the subventricular zone (SVZ) niche after brain injury in a Notchl-dependent manner [37]. Considering the contrasting results, further investigation on the role of THBS in TBIinduced cognitive impairments is required. Talin-1, which is the key talin member in immune cells, was found to be significantly upregulated in the TBI patients with cognitive impairments as compared to the negative and HC subjects. Previous studies have indicated that Talin-1 plays a role in tumour formation, migration, and metastasis in different types of cancer [38, 39]. However, the impact of Talin1 on TBI-induced cognitive impairments has yet to be unravelled.

Although the altered serum DEPs were identified and their possible underlying mechanisms were investigated, the present study has several limitations. First, only the serum of TBI patients with cognitive impairments was analysed. To more accurately reflect the pathophysiology of cognitive deficits following TBI, the plasma and CSF from the same individuals with post-TBI cognitive impairments should be analysed using iTRAQ-based quantitative proteomic approaches in the future. Second, the $5 \mathrm{DEPs}$ selected for ELISA validation are not brain specific. Moreover, a correlation between the 5 DEPs and the degree of cognitive impairment has not been established. Third, the present study included only a small number of patients; therefore, additional studies using a larger patient population should be conducted to fully confirm/validate the current findings. Fourth, similar to previous studies [40-42], we also only performed our experiment using one time point, which may result in overinterpretation of the findings. Additional time points would be beneficial for achieving optimal results. Lastly, the aim of this study was to identify the common underlying pathologies of TBI-related cognitive impairments, irrespective of the heterogeneity of the injuries sustained and the variability of the resulting cognitive deficits [43]. Thus, this study included TBI patients with different lesion locations, including those of the frontal lobe, temporal lobe, and parietal lobe, and we did not analytically distinguish these types of TBI patients.

\section{Conclusion}

To the best of our knowledge, the present study was the first to use an iTRAQ-based quantitative proteomic approach to identify DEPs in serum samples obtained from TBI patients with cognitive deficits to better understand the pathophysiology of cognitive impairments following TBI. Using an iTRAQ-based quantitative proteomic analysis, serum proteome alterations in patients with cognitive impairment after TBI were identified, and 56 DEPs were found to be specifically related to TBI-induced cognitive impairment. Moreover, bioinformatic analysis revealed that AD signaling pathways and lipid metabolism are involved in the pathophysiology of cognitive deficits following TBI. However, the limitations of the present study require further investigation and large-scale validation. 


\section{Competing Interests}

The authors declare that they have no competing interests.

\section{Authors' Contributions}

The work presented here was conducted in collaboration between all authors. Weijun Peng conceived and designed the work. Weijun Peng, Zhe Wang, and Xin-gui Xiong analysed the data, interpreted the results, and drafted the manuscript. Qinghua Liang, Wei Huang, Yang Wang, and $\mathrm{Zi}$-an Xia carried out the clinical research and participated in the acquisition, analysis, or interpretation of the data. Chuhu Zhang contributed to the data collection, analysed the data, and interpreted the results. All authors reviewed and approved the final manuscript. Weijun Peng, Zhe Wang, and $\mathrm{Zi}$-an Xia contributed equally to this study.

\section{Acknowledgments}

This work was supported by the Young Scientists Fund of the National Natural Science Foundation of China (Grant nos. 81102564, 81603670) and the National Natural Science Foundation of China (Grant no. 81373705). The authors also thank Gene Denovo Co. (Guangzhou, People's Republic of China) for technical support.

\section{References}

[1] M. M. Morawska, F. Büchele, C. G. Moreira, L. L. Imbach, D. Noain, and C. R. Baumann, "Sleep modulation alleviates axonal damage and cognitive decline after rodent traumatic brain injury," Journal of Neuroscience, vol. 36, no. 12, pp. 34223429, 2016.

[2] A. R. Rabinowitz and H. S. Levin, "Cognitive sequelae of traumatic brain injury," Psychiatric Clinics of North America, vol. 37, no. 1, pp. 1-11, 2014.

[3] I. Cristofori and H. S. Levin, "Traumatic brain injury and cognition," Handbook of Clinical Neurology, vol. 128, pp. 579611, 2015.

[4] K. R. Walker and G. Tesco, "Molecular mechanisms of cognitive dysfunction following traumatic brain injury," Frontiers in Aging Neuroscience, vol. 5, article 29, 2013.

[5] D. J. Titus, N. M. Wilson, J. E. Freund et al., "Chronic cognitive dysfunction after traumatic brain injury is improved with a phosphodiesterase 4B inhibitor," Journal of Neuroscience, vol. 36, no. 27, pp. 7095-7108, 2016.

[6] X. Wang, A. Li, Y. Guo et al., "iTRAQ-based proteomics screen identifies LIPOCALIN-2 (LCN-2) as a potential biomarker for colonic lateral-spreading tumors," Scientific Reports, vol. 6, Article ID 28600, 2016.

[7] M. Li, F. Peng, G. Li et al., "Proteomic analysis of stromal proteins in different stages of colorectal cancer establishes Tenascin-C as a stromal biomarker for colorectal cancer metastasis," Oncotarget, vol. 7, no. 24, pp. 37226-37237, 2016.

[8] J. Muenchhoff, A. Poljak, F. Song et al., "Plasma protein profiling of mild cognitive impairment and Alzheimer's disease across two independent cohorts," Journal of Alzheimer's Disease, vol. 43, no. 4, pp. 1355-1373, 2015.

[9] R. Sharma, H. Gowda, S. Chavan et al., "Proteomic signature of endothelial dysfunction identified in the serum of acute ischemic stroke patients by the iTRAQ-based LC-MS approach," Journal of Proteome Research, vol. 14, no. 6, pp. 24662479, 2015.

[10] P. Zhang, S. Zhu, Y. Li et al., "Quantitative proteomics analysis to identify diffuse axonal injury biomarkers in rats using iTRAQ coupled LC-MS/MS," Journal of Proteomics, vol. 133, pp. 93-99, 2016.

[11] P. Wu, Y. Zhao, S. J. Haidacher et al., "Detection of structural and metabolic changes in traumatically injured hippocampus by quantitative differential proteomics," Journal of Neurotrauma, vol. 30, no. 9, pp. 775-788, 2013.

[12] F. Crawford, G. Crynen, J. Reed et al., "Identification of plasma biomarkers of TBI outcome using proteomic approaches in an APOE mouse model," Journal of Neurotrauma, vol. 29, no. 2, pp. 246-260, 2012.

[13] L. Yi, S. Shi, Y. Wang et al., "Serum metabolic profiling reveals altered metabolic pathways in patients with post-traumatic cognitive impairments," Scientific Reports, vol. 6, article 21320, 2016.

[14] A. Norup, K. S. Kristensen, L. Siert, I. Poulsen, and E. L. Mortensen, "Neuropsychological support to relatives of patients with severe traumatic brain injury in the sub-acute phase," Neuropsychological Rehabilitation, vol. 21, no. 3, pp. 306-321, 2011.

[15] W. D. Gouvier, P. D. Blanton, K. K. LaPorte, and C. Nepomuceno, "Reliability and validity of the Disability Rating Scale and the Levels of Cognitive Functioning Scale in monitoring recovery from severe head injury," Archives of Physical Medicine and Rehabilitation, vol. 68, no. 2, pp. 94-97, 1987.

[16] N. M. A. White, O. Masui, L. V. DeSouza et al., "Quantitative proteomic analysis reveals potential diagnostic markers and pathways involved in pathogenesis of renal cell carcinoma," Oncotarget, vol. 5, no. 2, pp. 506-518, 2014.

[17] W. Huang, Q. Liang, J. Chen et al., "Quantitative proteomic analysis of synovial tissue from rats with collagen-induced arthritis," RSC Advances, vol. 5, no. 105, pp. 86088-86101, 2015.

[18] D.-D. Xu, D.-F. Deng, X. Li et al., "Discovery and identification of serum potential biomarkers for pulmonary tuberculosis using iTRAQ-coupled two-dimensional LC-MS/MS," Proteomics, vol. 14, no. 2-3, pp. 322-331, 2014.

[19] C. Wang, L. Wei, L. Shi et al., "Screening and identification of five serum proteins as novel potential biomarkers for cured pulmonary tuberculosis," Scientific Reports, vol. 5, Article ID 15615, 2015.

[20] H. Mi, S. Poudel, A. Muruganujan, J. T. Casagrande, and P. D. Thomas, "PANTHER version 10: expanded protein families and functions, and analysis tools," Nucleic Acids Research, vol. 44, no. 1, pp. D336-D342, 2016.

[21] D. Szklarczyk, A. Franceschini, S. Wyder et al., "STRING v10: protein-protein interaction networks, integrated over the tree of life," Nucleic Acids Research, vol. 43, no. 1, pp. D447-D452, 2015.

[22] Z. Xing, Z. Xia, W. Peng et al., "Xuefu Zhuyu decoction, a traditional Chinese medicine, provides neuroprotection in a rat model of traumatic brain injury via an anti-inflammatory pathway," Scientific Reports, vol. 6, Article ID 20040, 2016.

[23] T. M. Sivanandam and M. K. Thakur, "Traumatic brain injury: a risk factor for Alzheimer's disease," Neuroscience and Biobehavioral Reviews, vol. 36, no. 5, pp. 1376-1381, 2012.

[24] B. L. Plassman and J. Grafman, "Traumatic brain injury and late-life dementia," Handbook of Clinical Neurology, vol. 128, pp. 711-722, 2015. 
[25] N. El Kadmiri, I. Slassi, B. El Moutawakil et al., "Glyceraldehyde-3-phosphate dehydrogenase (GAPDH) and Alzheimer's disease," Pathologie Biologie, vol. 62, no. 6, pp. 333-336, 2014.

[26] M. R. Hara and S. H. Snyder, "Nitric oxide-GAPDH-Siah: a novel cell death cascade," Cellular and Molecular Neurobiology, vol. 26, no. 4-6, pp. 527-538, 2006.

[27] J. Huang, N. Xiong, C. Chen et al., "Glyceraldehyde-3phosphate dehydrogenase: activity inhibition and protein overexpression in rotenone models for Parkinson's disease," Neuroscience, vol. 192, pp. 598-608, 2011.

[28] D. H. O’Day and M. A. Myre, "Calmodulin-binding domains in Alzheimer's disease proteins: extending the calcium hypothesis," Biochemical and Biophysical Research Communications, vol. 320, no. 4, pp. 1051-1054, 2004.

[29] D. H. O’Day, K. Eshak, and M. A. Myre, "Calmodulin binding proteins and Alzheimer's disease," Journal of Alzheimer's Disease, vol. 46, no. 3, pp. 553-569, 2015.

[30] H. Yang, T. Zhou, H. Wang et al., "Lipoprotein lipase deficiency leads to $\alpha$-synuclein aggregation and ubiquitin C-terminal hydrolase L1 reduction," Neuroscience, vol. 290, pp. 1-10, 2015.

[31] X. Xian, T. Liu, J. Yu et al., "Presynaptic defects underlying impaired learning and memory function in lipoprotein Lipasedeficient mice," The Journal of Neuroscience, vol. 29, no. 14, pp. 4681-4685, 2009.

[32] H. Gong, W. Dong, S. W. Rostad et al., "Lipoprotein lipase (LPL) is associated with neurite pathology and its levels are markedly reduced in the dentate gyrus of Alzheimer's disease brains," Journal of Histochemistry and Cytochemistry, vol. 61, no. 12, pp. 857-868, 2013.

[33] S. K. Kunutsor, H. Khan, K. Nyyssönen, and J. A. Laukkanen, "Is lipoprotein (a) protective of dementia?" European Journal of Epidemiology, vol. 31, no. 11, pp. 1149-1152, 2016.

[34] M. J. A. Amar, T. Sakurai, A. Sakurai-Ikuta et al., "A novel apolipoprotein C-II mimetic peptide that activates lipoprotein lipase and decreases serum triglycerides in apolipoprotein Eknockout mice," Journal of Pharmacology and Experimental Therapeutics, vol. 352, no. 2, pp. 227-235, 2015.

[35] A. Arias-Vásquez, A. Isaacs, Y. S. Aulchenko et al., "The cholesteryl ester transfer protein (CETP) gene and the risk of Alzheimer's disease," Neurogenetics, vol. 8, no. 3, pp. 189-193, 2007.

[36] H. J. Yang, S. P. Ma, F. Ju et al., "Thrombospondin-4 promotes neuronal differentiation of NG2 cells via the ERK/MAPK pathway," Journal of Molecular Neuroscience, vol. 60, no. 4, pp. 517-524, 2016.

[37] E. J. Benner, D. Luciano, R. Jo et al., "Protective astrogenesis from the SVZ niche after injury is controlled by Notch modulator Thbs4," Nature, vol. 497, no. 7449, pp. 369-373, 2013.

[38] N. Xu, H.-J. Chen, S.-H. Chen et al., "Upregulation of Talin-1 expression associates with advanced pathological features and predicts lymph node metastases and biochemical recurrence of prostate cancer," Medicine, vol. 95, no. 29, Article ID e4326, 2016.

[39] Y.-F. Xu, X.-Y. Ren, Y.-Q. Li et al., "High expression of Talin-1 is associated with poor prognosis in patients with nasopharyngeal carcinoma," BMC Cancer, vol. 15, no. 1, article 332, 2015.

[40] X. Zhang, X. Yin, H. Yu et al., "Quantitative proteomic analysis of serum proteins in patients with Parkinson's disease using an isobaric tag for relative and absolute quantification labeling, two-dimensional liquid chromatography, and tandem mass spectrometry," Analyst, vol. 137, no. 2, pp. 490-495, 2012.
[41] Y. Zhan, Y.-T. Yang, H.-M. You et al., "Plasma-based proteomics reveals lipid metabolic and immunoregulatory dysregulation in post-stroke depression," European Psychiatry, vol. 29, no. 5, pp. 307-315, 2014.

[42] Q. Wang, X. Su, X. Jiang et al., "iTRAQ technology-based identification of human peripheral serum proteins associated with depression," Neuroscience, vol. 330, pp. 291-325, 2016.

[43] P. O. Jenkins, M. A. Mehta, and D. J. Sharp, "Catecholamines and cognition after traumatic brain injury," Brain, vol. 139, no. 9, pp. 2345-2371, 2016. 

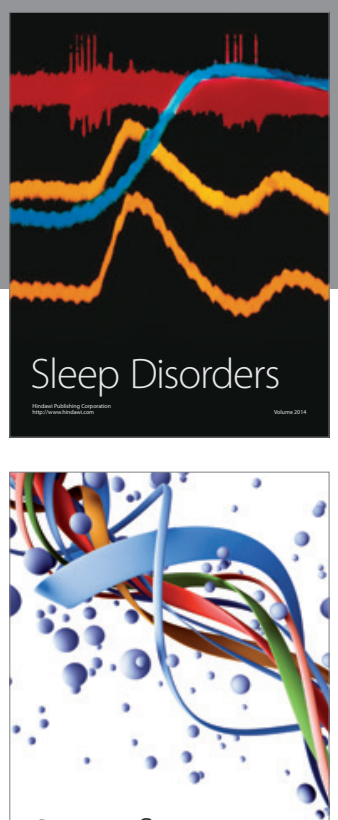

Scientifica
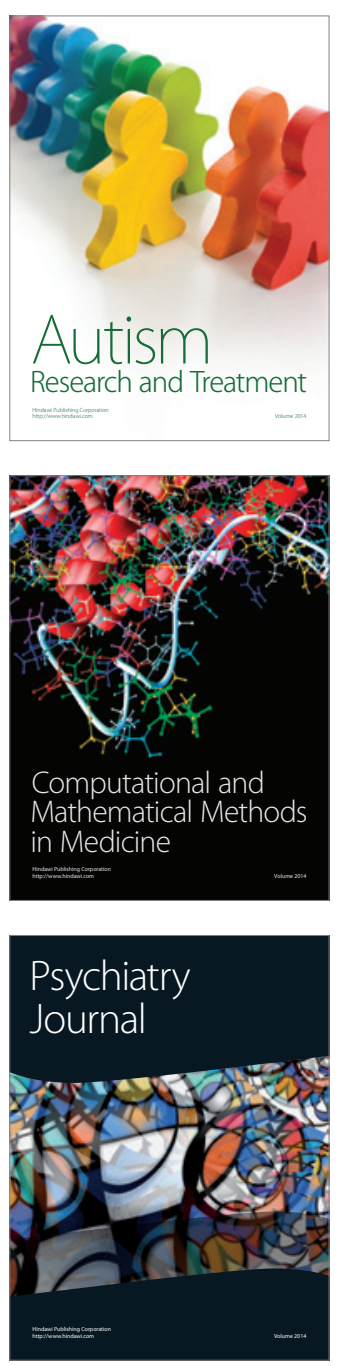
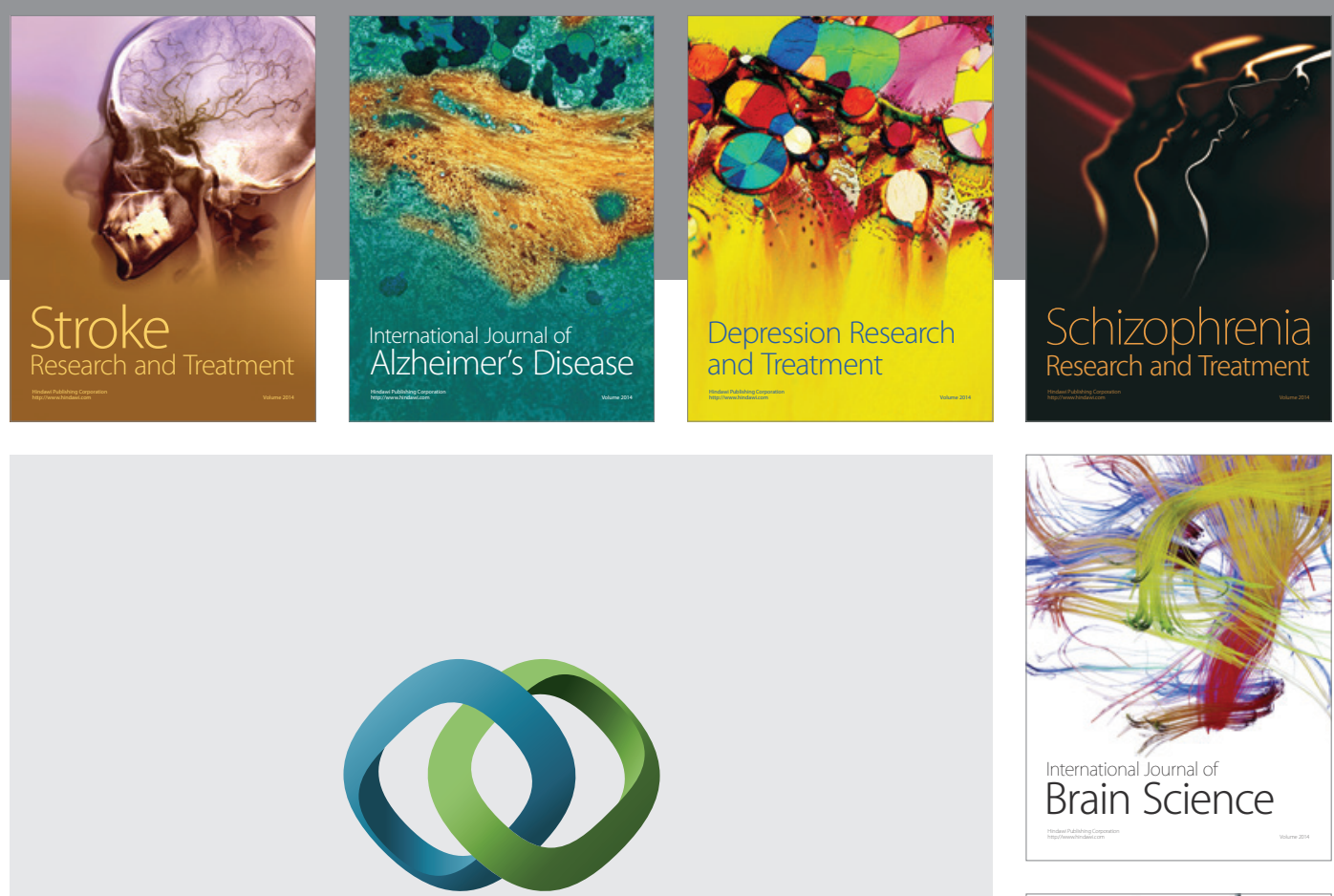

\section{Hindawi}

Submit your manuscripts at

https://www.hindawi.com
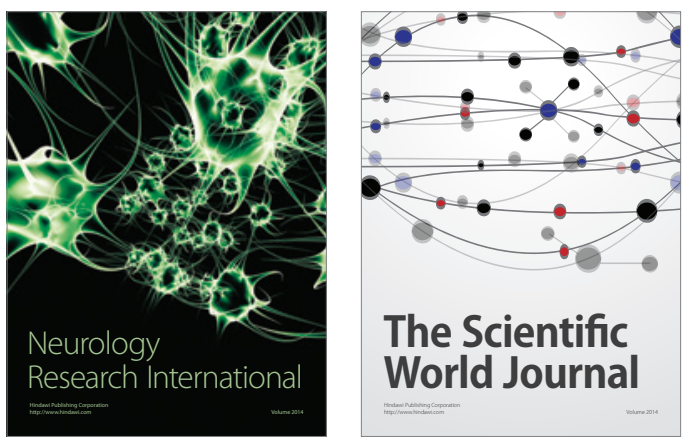

The Scientific World Journal

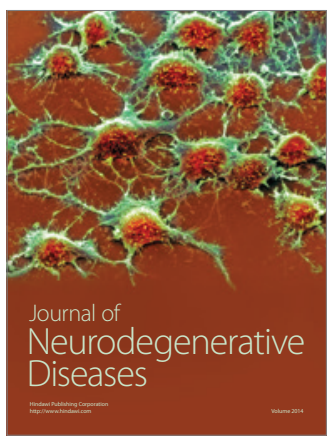

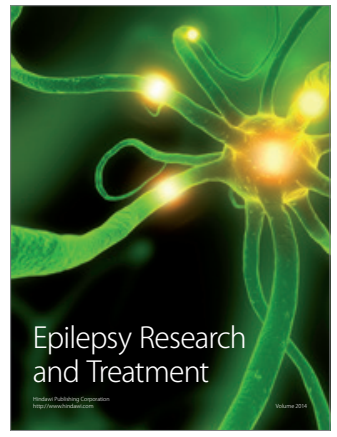

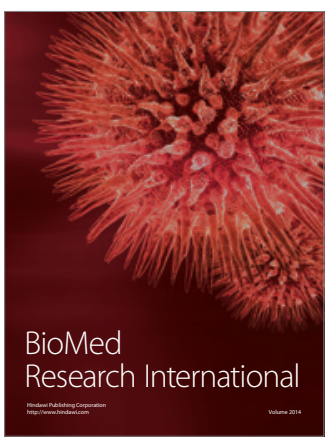

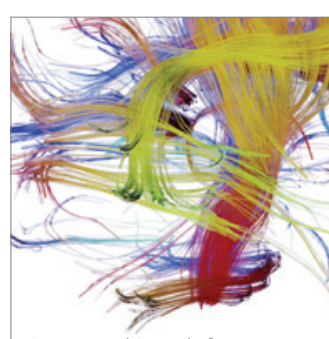

Brain Science

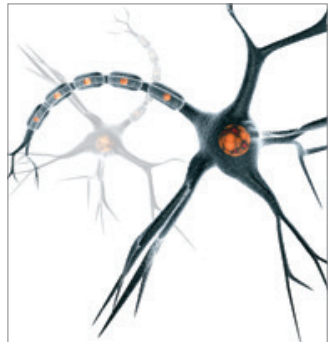

Neural Plasticity
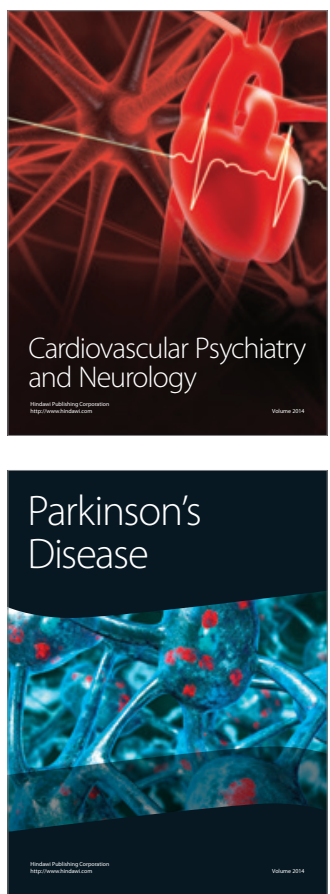